\title{
German Contributions to Mindfulness Research, Part 2: Assessment of Mindfulness
}

\author{
Zeno Kupper • Stefan Schmidt
}

Published online: 20 November 2012

(C) Springer Science+Business Media New York 2012

In the last three decades, mindfulness-based interventions and mindfulness as a general principle and practice have kindled a rapidly growing scientific interest. This interest has manifested in multiple forms and research programs according to the various traditions in philosophy, neuroscience, medicine, psychology, and other fields of science. One of these endeavors has been the quantitative assessment of "mindfulness" as a psychological state or trait. Utilizing the most common method of psychological assessment by questionnaires, various mindfulness questionnaires based on self-rating were developed. This enterprise has encountered great interest and adaption in empirical research. Nonetheless, mindfulness questionnaires have also encountered fundamental criticism, sometimes from researches who pioneered this field (Grossman 2008; Grossman and Van Dam 2011). Grossman (2008) identified six critical issues related to the self-rating of mindfulness: (1) conceptual difficulties; (2) potential biases of inventory developers (e.g., relatively modest level of experience in mindfulness practice and Buddhist psychological theory); (3) differences in semantic understanding of mindfulness items in respondents, depending on their level of experience with mindfulness practice; (4) potential discrepancies between self-ratings and "real mindfulness"; (5) biases in ratings stemming from long-term practice or the participation in mindfulness-based interventions and, as a consequence of these problems, (6) difficulties in the validation of mindfulness scales.

\footnotetext{
Z. Kupper $(\bowtie)$

Department of Psychotherapy, University Hospital of Psychiatry, University of Bern, Laupenstrasse 49,

3010 Bern, Switzerland

e-mail: zeno.kupper@spk.unibe.ch

S. Schmidt

Center for Meditation, Mindfulness and Neuroscience Research, University Medical Center Freiburg, Breisacher Str. 115b, 79106 Freiburg, Germany

e-mail: stefan.schmidt@uniklinik-freiburg.de
}

Grossman's vigorous critique makes it obvious that the assessment of self-attributed mindfulness is a non-trivial or even difficult undertaking. It is beyond the scope of this editorial to discuss these issues in detail and even more so to discuss possible solutions (cf. Baer 2011). Just as a note, we agree that the proper clarification of the context of any assessment of self-attributed mindfulness is of great importance (Schmidt and Kupper 2012). It is also related to this view that we encouraged the authors of these two issues to write more precisely of the assessment of self-attributed mindfulness since this comes closer to the process taking place when filling in a questionnaire. We share Grossman's concerns regarding an uninformed use of mindfulness scales in psychological research. However, whereas these issues are in fact critical, rather than dismissing the assessment of mindfulness-related variables, one could take these points as an encouragement for further insights and creative developments in mindfulness research. We would like to assume that there are even some specific factors, related to the topic of mindfulness itself, which could support such progress in mindfulness research. To name two, we assume that a strong foundation of mindfulness research in personal experience of mindfulness practice as well as attitudes and virtues arising from such practice may be of great benefit for mindfulness research. Borrowing words the renowned Vietnamese Buddhist teacher Thich Nhat Hanh likes to use for life in general, mindfulness researchers should be very aware that scientific "flowers" (prominent results and currently flourishing research approaches) are on the way to the garbage, and that "garbage" can turn into flowers very soon.

This Special Issue includes part 2 of contributions from a conference on clinical mindfulness research which took place in June 2011 in Bern, Switzerland, organized by an informal network of mindfulness researchers in German-speaking countries and the University Hospital of Psychiatry Bern, Department of Psychotherapy (Prof. Dr. W. Tschacher), and 
the Clinical Psychology Services (Prof. Dr. F. Moggi). Part 1 focused on the context and concept of mindfulness and part 2 concentrates on measuring mindfulness. The assessment of self-attributed mindfulness was a central topic at the Bern 2011 conference. The contributions addressing the selfrating of mindfulness in this issue aim at addressing some of the critical issues listed by Grossmann (2008) in an empirical approach. In a first contribution, Sauer et al. present a theoretical overview of existing scales, identify conceptual differences between the instruments, and provide suggestions for a more comprehensive assessment of mindfulness. As a background for their analysis, Sauer et al. also report informative statistics regarding the use of mindfulness scales in current research. Furthermore, they provide suggestions for the use of instruments depending on specific research contexts. Bergomi et al. describe initial steps in the development and empirical study of a scale aiming at a comprehensive assessment of selfattributed mindfulness. The preliminary version of their scale, the CHIME- $\beta$, includes and integrates aspects that were identified through an overview of eight validated scales. The study provides interesting exploratory results related to two main issues: the conceptual coverage (and thus content validity) of mindfulness scales and the influence of mindfulness practice on the interrelationships among aspects of mindfulness. Two further contributions explore properties of the classical and pioneering Freiburg Mindfulness Inventory (FMI; Walach et al. 2006). Belzer et al. assessed the cognitive processes in participants while filling in the FMI by an innovative qualitative approach. They found that in fact some items may be difficult to understand for persons without meditation experience, which could endanger the construct validity of the FMI in meditation-naïve subjects. In an innovative psychometric analysis, Sauer et al. explored properties of the short form of the FMI in a large nonclinical sample. In contrast to some older work proposing one general mindfulness factor in the FMI, their results suggest using two factors of "presence" and "acceptance." The issue concludes with a qualitative and a theoretical contribution. Full et al. report and analyze remarkable data from highly experienced Buddhist meditators regarding alterations in perception due to longterm meditation practice. Finally, Blaser proposes a "spatial attention" framework for the integration of mindfulnessrelated and self-related constructs.

\section{References}

Baer, R. A. (2011). Measuring mindfulness. Contemporary Buddhism, $12,241-261$.

Walach, H., Buchheld, N., Buttenmüller, V., Kleinknecht, N., \& Schmidt, S. (2006). Measuring mindfulness - the Freiburg Mindfulness Inventory (FMI). Personality and Individual Differences, 40, 1543-1555.

Grossman, P. (2008). On measuring mindfulness in psychosomatic and psychological research. Journal of Psychosomatic Research, 64, 405-408.

Grossman, P., \& Van Dam, N. T. (2011). Mindfulness, by any other name. .. : trials and tribulations of sati in western psychology and science. Contemporary Buddhism, 12, 219-223.

Schmidt, S., \& Kupper, Z. (2012). German contributions to mindfulness research, part 1: context and concept of mindfulness. Mindfulness, 3, 171-173. 